\title{
Role Of Various Factors In The Treatment Of Optic Neuritis----A Study
}

\author{
Dr Rajendra Prasad.J ${ }^{1}$, Dr Ravikiran $\mathrm{P}^{2}$ \\ ${ }^{1}$ Associate Professor ${ }^{2}$ Associate Professor Dept of Ophthalmology, \\ Dr PSIMS\&RF, Chinoutpally, India
}

\begin{abstract}
Aim: To analyse the influence of various factors on the visual outcome of Optic neuritis patients

Materials \& Methods: A total of 116 patients with optic neuritis were studied as a prospective study for two years in the department of Ophthalmology, Dr PSIMS\&RF, Chinoutpalli. All cases were investigated for visual acuity, colour vision, APD, visual fields. At the end of the study all the above parameters were recorded and analysed.

Discussion: All the patients having optic neuritis were presented with defective vision[100\%].70 cases had ocular pain[60.34\%].32 patients had defective colour vision[27.58\%].28 patients had defective visual fields[24.14\%].The study showed a female preponderance with $64 \%$ of cases. History of recurrence was noted in $10 \%$ of cases.

Conclusion: In our study no specific factor is associated with the aetiology of optic neuritis. $18 \%$ of cases showed bilaterality. After treatment[ONTT],all the cases showed significant improvement in the visual acuity. Colour vision was improved at the end of third month of treatment. ONTT regime has a very significant role in the treatment of Optic neuritis cases.
\end{abstract}

Key words: optic neuritis, visual acuity, prednisolone, optic neuritis treatment trial

\section{Introduction}

Optic neuritis is a term used to refer to inflammation of the optic nerve. When it is associated with a swollen optic disc, it is called papillitis or anterior optic neuritis ${ }^{1}$. When the optic disc appears normal, the term retrobulbar optic neuritis or retrobulbar neuritis are used. In the absence of signs of multiple sclerosis or other systemic disease, the term optic neuritis refers to isolated, monosymptomatic, or idiopathic. It is likely that most cases of isolated acute optic neuritis are a forme fruste of multiple sclerosis. Patients in whom optic neuritis occurs as an isolated phenomenon have a higher risk of developing multiple sclerosis at a later date than the normal population. Optic neuritis is also a part of the demyelinating syndrome called neuromyelitis optica or "Devic's disease".

Incidence of optic neuritis in several studies ranges from one to six new cases per year per one lakh population, Patients with optic neuritis are typically young with a peak incidence in the third and fourth decade. Women are more commonly affected than men. Optic neuritis is characterised by sudden partial or complete loss of vision, loss of colour vision, pain on movement of the affected eye, afferent pupillary defect, normal or swollen optic nerve head and central visual field defects.

Optic neuritis can spontaneously recover or can lead to permanent optic nerve damage leading to permanent visual loss. Spontaneous recovery can be speeded up by parenteral steroids (Optic Neuritis Treatment Trial) at appropriate time. Permanent optic nerve damage can be prevented by this.

\section{Optic Neuritis}

The term optic neuritis refers to inflammatory optic neuropathy that accompanies demyelinating disease. When it is associated with swollen optic disc, it is called papillitis or anterior optic neuritis. When the optic disc appears normal, the terms retrobulbar optic neuritis or retrobulbar neuritis are used. In the absence of signs of multiple sclerosis (MS) or other systemic disease the optic neuritis is referred to as isolated, monosymptomatic, or idiopathic. The pathogenesis of isolated optic neuritis is presumed to be demyelination of the optic nerve, similar to that seen in multiple sclerosis. It is likely that most cases of isolated acute optic neuritis are a "forme fruste" of $\mathrm{MS}^{2}$.

Optic neuritis can be caused by disorders other than MS and related demyelinating diseases. In addition, two unusual variants of optic neuritis can occur in some patients. "Neuroretinitis" is a term used to describe inflammatory involvement of both the intraocular optic nerve and the peripapillary retina. They show optic disc swelling, extensive retinal edema, haemorrhages, and hard exudates (Hpid) in the macula in a starshaped pattern. Optic perineuritis also called perioptic neuritis describes inflammatory involvement of the optic 
nerve sheaths without inflammation of the nerve itself associated with optic disc swelling that is unassociated with visual complaints.

\section{Symptoms of optic neuritis:}

The major symptoms in patients with acute optic neuritis are loss of central vision and pain in and around the affected eye.

1. Loss of central vision

2. Loss of visual field

3. Ocular or Orbital pain

4. Positive visual phenomenon

\section{Optic Neuritis Treatment Trial (Ontt)}

The optic neuritis treatment trial is a multicenter controlled clinical trial that was funded by the National Eye Institute of the National Institutes of Health I in the United States. In this trial 455 patients with acute unilateral optic neuritis were enrolled. The primary objective of the trial was the assessment of the efficacy of corticosteroids in the treatment of optic neuritis. It also gives some information about the clinical profile of optic neuritis, its natural history and its relationship to $\mathrm{MS}^{3}$.

Inclusion criteria in ONTT included a clinical syndrome consistent with unilateral optic neuritis (including a relative afferent pupillary defect and a visual field defect in the affected eye), visual symptoms of eight days or less, no previous episodes of optic neuritis in the affected eye, no previous corticosteroid treatment for optic neuritis or MS, and no evidence of a systemic disease other than MS as a cause for the optic neuritis ${ }^{4}$.

Patients were assigned to one of three treatment groups:

1. Oral prednisone $(1 \mathrm{mg} / \mathrm{kg} /$ day $)$ for 14 days

2. Intravenous methyl prednisolone sodium succinate $(250 \mathrm{mg}$ QID for three days) followed by oral prednisone $(1 \mathrm{mg} / \mathrm{kg} /$ day $)$ for 11 days

3. Oral placebo for 14 days

\section{Methods of examination:}

Rate of visual recovery and visual outcome were assessed by

1. Snellen acuity with a retroilluminated Bailey-Lovie chart at four meters

2. Colour vision with the Farnsworth-Munsell 100-hue test ${ }^{5}$

3. Contrast sensitivity with the Pelli-Robson chart

4. Perimetry with the Humphrey Field Analzer (program 30-2) and Goldmann perimeter.

Complete neurological examination was performed at study entry, after six months, after one year, and then yearly. Clinically definite MS was diagnosed when a patient develops new neurological symptoms attributable to demyelination in one or more regions of the central nervous system, other than new optic neuritis in either eye, occurring atleast four weeks after the optic neuritis at study entry and lasting more than 24 hours with abnormalities documented on neurologic examination.

\section{Observation of ONTT:}

1. Most patients in all three treatment groups had a good recovery of vision.

2. After six months of follow up, the median visual acuity in each age group was $20 / 60$ and less than $10 \%$ of the patients in each group had a visual acuity of 20/50 or worse.

3. One year after the onset of visual symptoms, there was no significant difference in mean visual cuity, color vision, contrast sensitivity, or visual field.

4. Patients treated with the regimen intravenous methyl prednisolone followed by oral prednisolone recovered vision considerably faster than patients treated with oral placebo.

5. The benefit of this treatment regimen was greatest in the first 15 days of follow up and decreased subsequently.

6. Patients treated with oral prednisone alone had an increased rate of recurrent attacks of optic neuritis in the previously affected eye and an increased rate of new attacks of optic neuritis in the fellow eye compared with patients in the other two groups. Oral Prednisolone in a dose of $1 \mathrm{mg} / \mathrm{kg} /$ day did not speed recovery of vision compared with no treatment, and did not improve ultimate visual acuity, compared with no treatment. It produced a higher rate of recurrence and new attacks of optic neuritis than no treatment ${ }^{5}$.

7. Patients treated with intravenous followed by oral corticosteroids regimen had a reduced rate of development of clinically definite multiple sclerosis during the first two years. The clinical benefit of the intravenous treatment lessened over time such that at three years of follow up, there was no significant difference in the rate of development of MS among the treatment groups. 
8. Benefit of the treatment was only seen in patients who had significantly abnormal brain MRI at the time of onset of the optic neuritis.

9. Ultimately the group concluded that there is no treatment for acute demyelinating optic neuritis that can improve the ultimate the visual prognosis compared with the natural history of the disorder'.

10. Recurrent attacks of optic neuritis were reported within five years in $20 \%$ patients in ONTT. The likelihood of visual acuity returning to normal decreases with each recurrence.

\subsection{Subject Selection:}

\section{Materials And Methods}

This is a prospective study. All the patients diagnosed as optic neuritis in the department of Ophthalmology, Dr PSIMS\&RF during August 2013 to July 2015 were included in the study.

\subsection{Inclusion criteria:}

All patients diagnosed as optic neuritis were studied.

\subsection{Exclusion criteria:}

The following patients were excluded from the study

1) Patients with Anterior Ischemic optic neuropathy

2) Patients with traumatic optic neuropathy

\subsection{Methods:}

In patients attending Dr PSIMS\&RF diagnosed as optic neuritis

1) Various risk factors associated with causation of optic neuritis were assessed.

2) Time of onset of symptoms \& time when the patient presented to hospital was noted.

3) In all these patients, visual function was evaluated by Snellen's chart for visual acuity, colour vision by Ishihara pseudoisochromatic chart, assessment of contrast sensitivity Peli Robson chart and fields were tested manually by Bjerrum's screen. Pupillary assessment was done and fundus examination was done using direct ophthalmoscopy.

4) Systemic Evaluation was done for diseases causing optic neuritis.

5) The patients were started on corticosteroids as in ONTT, 3 days of I.V Methyl prednisolone at a dose of $500 \mathrm{mg}$ twice daily followed by Tab.Prednisolone $\mathrm{Img} / \mathrm{kg} /$ day for 11 days and then a short taper.

6) If there were any contraindications for starting steroids, physician opinion was obtained.

7) They were also supplemented with inj.vit B1B6B12 on alternate days.

8) Related investigations were done.

9) Vision, pupil, field, colour vision, contrast sensitivity and fundus were assessed after treatment.

10) Follow up visits were given two weeks, one month and three months after the initial discharge.

11) Analysis of aetiology and visual outcome of recurrent optic neuritis was done.

\subsection{Screening procedures}

1) Vision, Pupillary assessment, Fundus, Fields, Colour vision, contrast sensitivity.

2) B Scan, VEP, MRI Brain and orbit as needed

\subsection{Follow up procedures/visits}

Follow up visits were given two weeks, one month and three months after the initial discharge. During each follow up assessment of vision, pupil, fundus, fields, contrast sensitivity and colour vision were done.

\subsection{Assessment Of Parameters}

Visual acuity, Pupillary assessment (Afferent pupillary defect), Fields (Central field defect) Colour vision (dyschromatopsia disproportionate to visual loss), contrast sensitivity, Fundus changes (swollen optic nerve head) and MRI (in case of multiple sclerosis).

Statistical analysis of visual outcome of optic neuritis was done in relation to the risk factors and treatment given.

\section{Observation And Results}

Totally 116 patients with acute optic neuritis were included in the study. This included first episode, recurrent episode, unilateral and bilateral cases. There were 94 unilateral patients and 22 bilateral patients were studied. Totally 138 eyes with optic neuritis were studied.

\section{Age Distribution}

Table 1 Age distribution of the study patients

\begin{tabular}{|l|l|l|}
\hline & No. Of patients & \% of total \\
\hline$<\mathbf{1 2}$ years & 12 & $10.34 \%$ \\
\hline $\mathbf{1 2}-18$ years & 7 & $6.05 \%$ \\
\hline
\end{tabular}


Role Of Various Factors In The Treatment Of Optic Neuritis----A Study

\begin{tabular}{|l|l|l|}
\hline $\mathbf{1 9 - 3 0}$ years & 22 & $18.96 \%$ \\
\hline $\mathbf{3 1 - 5 0}$ yrs & 57 & $49.14 \%$ \\
\hline$>\mathbf{5 0}$ yrs & 18 & $15.51 \%$ \\
\hline
\end{tabular}

Of the total 116 patients 12 were under the age group of $<12$ yrs (10.34\%). Seven (6.05\%) patients belong to the age group of 13 -- 18 years of age. 22 (18.96\%) patients were in the age group of 19-30 yrs, 57 $(49.14 \%)$ in $31-50$ yrs and $18(15.51 \%)$ patients were $>50$ yrs of age. (Figure 1) Majority of the patients come under the age group of $31-50 \mathrm{yrs}$ of age. Less number of patients were in the age group of 13- 18 yrs of age group. (Table 1)

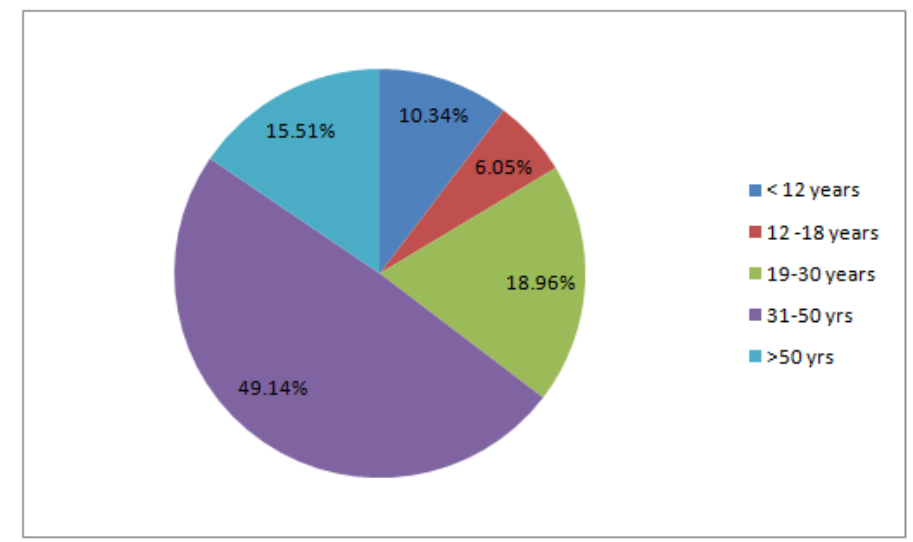

Figure 1 : Age distribution

Sex distribution:

Table 2 Sex distribution

\begin{tabular}{|l|l|l|}
\hline & No. Of patients & \% of total \\
\hline Male & 42 & $36.21 \%$ \\
\hline Female & 74 & $63,79 \%$ \\
\hline Total & 116 & 100 \\
\hline
\end{tabular}

Totally there were 116 patients of which 74 females $(63,79 \%)$ were affected. Males were about 42 in number $(36.21 \%)$, (Table 2) So females were commonly affected. (Figure 2)

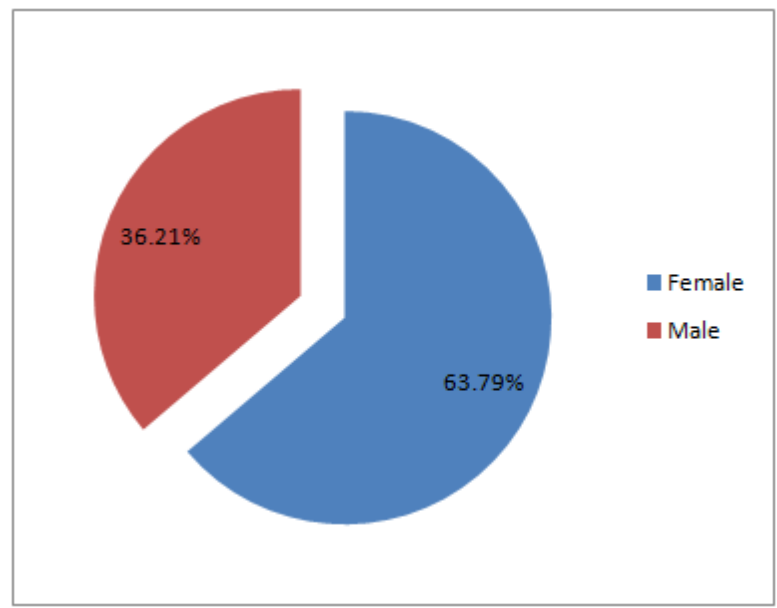

Figure 2 : Sex distribution

\section{Complaints}

Table 4 Complaints of the patients

\begin{tabular}{|l|l|l|}
\hline & No. Of patients & \% of total \\
\hline Defective vision & 116 & 100 \\
\hline Ocular pain & 70 & 60.34 \\
\hline Defective colour vision & 32 & 27.58 \\
\hline Defective field of vision & 28 & 24.14 \\
\hline
\end{tabular}


All the patients presented with defective vision(100\%). (Figure 4) 70 patients had ocular pain $(60.34 \%)$, Defective colour vision was present in 32 patients $(27.58 \%), 28$ patients had defective field of vision. (24.14\%), (Table 4)

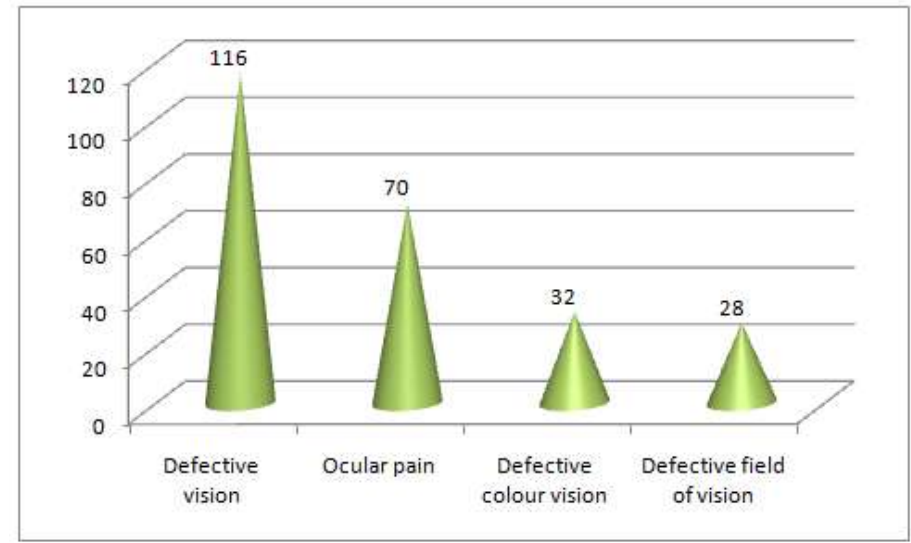

Figure 3 : Complaints of the Patients

\section{Recurrent Attack}

Table 5 Recurrent cases of optic neuritis

\begin{tabular}{|l|l|l|}
\hline & No. of cases & \% of total \\
\hline First episode & 104 & 89.66 \\
\hline Second episode & 12 & 10.34 \\
\hline
\end{tabular}

Of the total 116 acute episode of optic neuritis, 12 patients had history of previous attack (10.34\%), (Figure 5) Of these 12, 8 gave history of treatment taken $(66,67 \%$ of recurrence) and 4 patients had not taken treatment $(33,34 \%)$ (Table 5) Of the 12 recurrent cases, 7 patient had attack in the same eye itself $(58.33 \%)$ and 5 had attack in the other eye (41.67\%) (Figure 6).

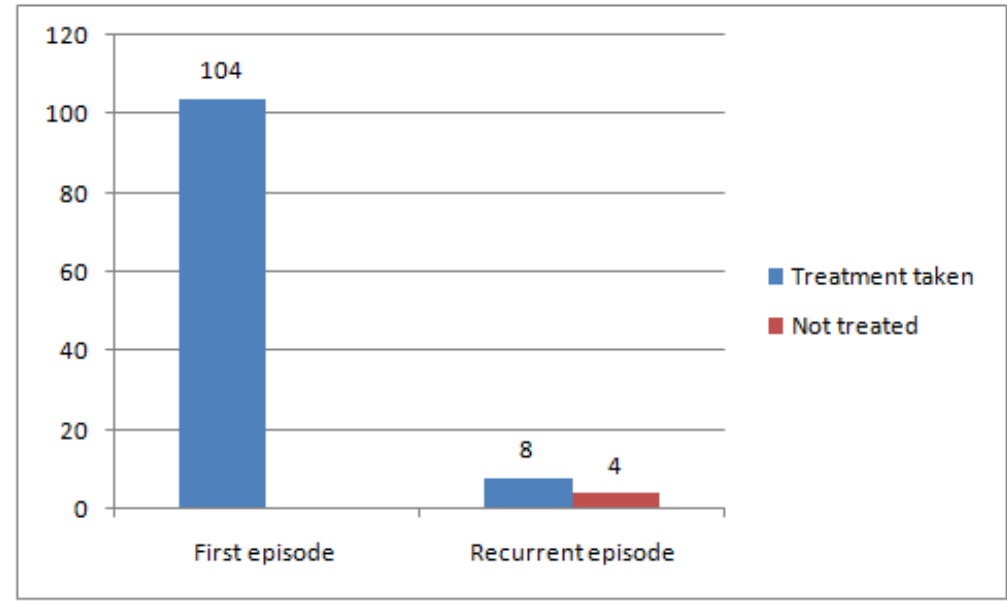

Figure 4 : Recurrent case of optic neuritis 


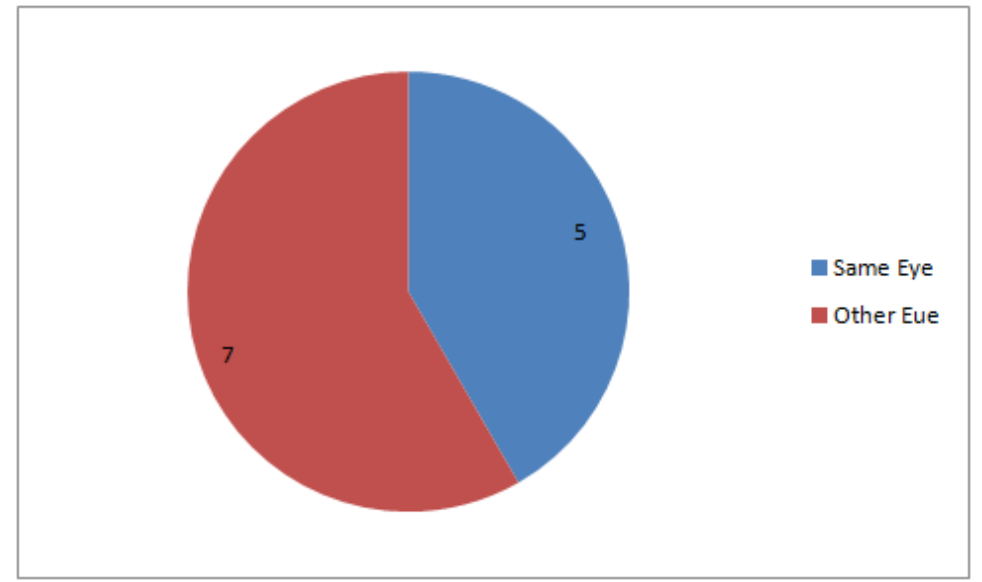

Figure 5 : Pattern of Recurrence

\section{Discussion}

All cases of optic neuritis reporting to our hospital during a period of two years were taken for study to assess the efficacy of treatment on various visual parameters including visual acuity, colour vision, pupil, fundus and fields. Analysis was done as regards to association of any specific risk factors with the final visual outcome.

In this study, totally 116 patients with 138 eyes affected with optic neuritis were studied. Among these, $50 \%$ of the patients were in the age group of 30 - 50 years which was also noted in the ONTT study. Only 6\% was reported in the age group of $12-18$ yrs.

$64 \%$ of cases were females indicating a female preponderance. This female preponderance persisted through all age groups.

Defective vision was reported in $100 \%$ patients. Defective colour vision was reported in $30 \%$. Field defects were complained in $28 \%$ of the patients. Although these complaints were co-existent with visual defect, the probable cfause of this might be poor visual acuity so that colour vision and fields could not be complained of by these patients.

History of recurrence i.e. previous attack of sudden loss of vision which improved later with or without treatment was present in $10 \%$ of individuals. Approximate intervals between recurrences was 2 years in this study. History of treatment was present in $65 \%$ of recurrent cases. But there was no difference in interval between two attack noted between the two groups (with or without treatment).

Recurrence was more common in the same eye (58\%) compared to recurrence in other eye.

Considering the past history, history of fever was present in $6 \%$ of patients. Fever was more common in children. History of treatment taken for tuberculosis was present in one patient. There was no history of trauma or recent vaccination.

MRI was advised in atypical cases. It was done in seven patients in whom 4 were normal. Two patients showed signs of demyelination. One patient showed feature of optic neuritis. MRI showing demyelination in the periventricular region is a strong predictor of future development of multiple sclerosis.

Most of the cases were unilateral in $80 \%$ of patients. Bilateral cases were common in children. History of fever was more common in children which were suggestive of possibility of viral etiology for optic neuritis.

Visual acuity at the time of presentation was $6 / 36-6 / 18$ in $48 \%$ of patients. Only $4 \%$ had PL vision. Colour vision was defective in $75 \%$ of patients at the time of presentation. Contrast sensitivity and fields were defective in all patients who had vision enough to be tested. Central scotoma was present in $42 \%$ of patients and non specific defects were noted in $45 \%$ of patients. During follow up fields were tested by Bjerrum's screen. Those who had abnormal field were subjected to automated perimetry at three months all of whom showed persistent field defect. . Abnormal pupillary reaction was noted in all the patients. Even after treatment pupillary abnormality persisted in $25 \%$ of patients. Retro bulbar neuritis was noted in $28 \%$ of the patients. Fundus abnormalities were resolved by three months in all patients. $8 \%$ of patients developed temporal pallor at three months of follow up indicating some residual damage to optic nerve.

After ONTT, visual acuity abnormalities resolved in $76 \%$ of patients by one month and $94 \%$ in three months. Colour vision and contrast sensitivity abnormality persisted even after three months.

ONTT hastened improvement of visual acuity in all the patients with optic neuritis.

\section{Conclusion}

- Females were predominantly affected than males in the ratio of 3:1.

- Most common affected patients were in the age group of 30-50 yrs.

- Recurrent cases included $10 \%$ of the patients. Recurrence was more common in the same eye. 
- No specific association with any etiological factor was noted.

- Almost all patients had defective vision. Around 25\% had complaints of defective colour vision, defective field of vision and $60 \%$ had ocular pain.

- Bilateral cases were around $18 \%$. They were more common in children.

- Fever was more common in children and more commonly associated with bilateral optic neuritis.

- Visual acuity at the time of presentation was 6/60 - 6/36. In all the patients pupillary reaction was abnormal. Colour vision was abnormal in $75 \%$ of patients. Contrast sensitivity was abnormal in all patients.

- Field defect was always noted in all patients most common was central scotoma.

- Retrobulbar neuritis was noted in $28 \%$ of patients. Other patients had optic neuritis. Most commonly disc oedema was noted. Rarely haemorrhage was noted.

- After ONTT almost all patients showed improvement in vision. This improvement of vision is statistically significant.

- Colour vision recovery was noted in $46 \%$ after three months of follow up.

- Fields showed persistent field defect after three months in the patients.

- Institution of ONTT has definite role in speeding up of recovery.

\section{References}

[1]. Fine B, Yanoff M : The optic nerve. Ocular Histology : A Text and Atlas, $2^{\text {nd }}$ ed., pp 272-287. Hagerstown, MD, Harper \& Row, 1979.

[2]. Optic Neurtis Study Group. Visual function 15 years after optic neuritis : a final follow-up report from the Optic Neuritis TreatmentTrial. Ophthalmology 2008; 115:1079-1082.

[3]. Kidd D: Inflammatory optic neuropathies not associated with multiple sclerosis. In : Neuro ophthalmology (Kidd DP, Newman NJ, Biousse V, eds). Boston : Butterworth Heineman 2008; pp 153-19.

[4]. American Academy of Ophthalmology Neurophthalmology eighteenth edition-pathogenesis of optic neuritis.

[5]. Hickman SJ et al. Optic Neurities : An Update Typical and Atypical Optic Neuritis. Neuro-Ophthalmology 2008; 32:237-248

[6]. Wilejto M, Shroff M, Buncic JR, et al; The clinical features, MRI findings, and outcome of optic neuritis in children. Neurology. 2006 Jul 25;67(2):258-62. 